\title{
Genomic Contingencies and the Potential for Local Adaptation in a Hybrid Species
}

\author{
Anna Runemark, ${ }^{1,2,}$ Laura Piñeiro Fernández, ${ }^{3}$ Fabrice Eroukhmanoff, ${ }^{1}$ and Glenn-Peter Sætre ${ }^{1}$ \\ 1. Centre for Ecological and Evolutionary Synthesis, Department of Biosciences, University of Oslo; 2. Department of Biology, \\ Lund University; 3. Institut für Systematische und Evolutionäre Botanik, University of Zürich \\ Submitted December 8, 2017; Accepted February 14, 2018; Electronically published April 23, 2018 \\ Online enhancements: appendix, supplemental material. Dryad data: https://dx.doi.org/10.5061/dryad.615s9q5.
}

\begin{abstract}
A BSTRACT: Hybridization is increasingly recognized as a potent evolutionary force. Although additive genetic variation and novel combinations of parental genes theoretically increase the potential for hybrid species to adapt, few empirical studies have investigated the adaptive potential within a hybrid species. Here, we address whether genomic contingencies, adaptation to climate, or diet best explain divergence in beak morphology using genomically diverged island populations of the homoploid hybrid Italian sparrow Passer italiae from Crete, Corsica, and Sicily. Populations vary significantly in beak morphology both between and within islands of origin. Temperature seasonality best explains population divergence in beak size. Interestingly, beak shape along all significant dimensions of variation was best explained by annual precipitation, genomic composition, and their interaction, suggesting a role for contingencies. Moreover, beak shape similarity to a parent species correlates with proportion of the genome inherited from that species, consistent with the presence of contingencies. In conclusion, adaptation to local conditions and genomic contingencies arising from putatively independent hybridization events jointly explain beak morphology in the Italian sparrow. Hence, hybridization may induce contingencies and restrict evolution in certain directions dependent on the genetic background.
\end{abstract}

Keywords: adaptation, beak shape, climate, diet, genetic constraints, Passer italiae.

\section{Introduction}

Adaptation to divergent ecological niches is a major factor in population divergence and speciation (Schluter 2000; Grant and Grant 2008; Schluter 2009). Adaptation in key traits where novel morphologies can allow for the invasion of new niches (Dumont et al. 2012) are of particular interest because divergence in these can drive speciation (Hunter 1998). In birds, key traits can also enable coexistence with closely related species (Miraldo and Hanski 2014) and hence spur

\footnotetext{
* Corresponding author; email: anna.runemark@ibv.uio.no. ORCIDs: Runemark, http://orcid.org/0000-0002-8976-5530; Piñeiro Fernández, http://orcid.org/0000-0002-8617-6490.
}

Am. Nat. 2018. Vol. 192, pp. 10-22. (C) 2018 by The University of Chicago. 0003-0147/2018/19201-58127\$15.00. All rights reserved. DOI: $10.1086 / 697563$ adaptive radiations and promote diversification (Schluter 2000; Jarvis et al. 2014). The beak is such a key trait, since beak shape adaptations have significantly contributed to the niche diversity in birds (Mallarino et al. 2012). Variation in beak size and shape is important for both feeding efficiency (Benkman 2002, 2016) and thermoregulation (Symonds and Tattersall 2010). It also affects song (Derryberry et al. 2012) and can hence be a target of sexual selection (Huber and Podos 2006). A classical example of beak morphology adaptation is the radiation of Darwin's finches on the Galapagos Islands, where divergent selection between groups of birds with different dietary preferences has caused a dramatic beak shape diversity (Grant and Grant 2006). Interestingly, hybridization can also generate new beak shapes that allow more efficient use of specific dietary resources (Grant and Grant 1996; Lamichhaney et al. 2015, 2016).

Hybridization is increasingly recognized as an important source of novel genetic variation (Mallet 2005, 2007; Abbott et al. 2013). It can spur novel adaptations by increasing genomic diversity and through changing the constraints on the direction of evolution (Selz et al. 2013; Lucek et al. 2016). Hybrids are expected to have more additive genetic variation than the parental species' genomes, and this increase is highest when the parent species are fixed for different alleles at each locus (Bailey et al. 2013; Eroukhmanoff et al. 2013a; Seehausen 2013). Furthermore, the mosaic genome from the combination of the two parental genome complexes (Rieseberg 2003) can give rise to phenotypes that are either intermediate or mosaic versions of the parents or transgressive phenotypes, which are beyond the range of the parental species (Rieseberg et al. 1999; Dittrich-Reed and Fitzpatrick 2012). The increase in additive genetic variation and the novel combinations of parental genes may increase the potential for hybrids to adapt (Rieseberg 2003; Eroukhmanoff et al. 2013a). Interestingly, different hybrid populations can attain strongly divergent genomic composition (Roy et al. 2015; Runemark et al. 2018b). However, hybrid species can also be subjected to contingencies resulting from mosaic patterns of parental inheritance (Eroukhmanoff et al. 
2013a). For instance, the allele frequency change for a trait with selection for alleles of one parent species may depend on the genome-wide proportion of alleles from that parent species. Hybrid genomes are under selection to purge incompatible allele combinations (Eroukhmanoff et al. 2017), and this could lead to selection for backcrossing toward one parent species (Baack and Rieseberg 2007). Under a scenario where multiple sites across the genome are selected to be compatible with a majority of alleles from the one parent species, the possibilities to increase the frequency of an allele from the other parent species in to response to selection for a trait value similar to that of the other parent species may be constrained. Here, we refer to this pattern as a genomic contingency (cf. Eroukhmanoff et al. 2013a). Tightly linked loci-for instance, those linked to an inversion-are not likely to be affected by recombination following hybridization, and for such pairs of loci parental allele combinations would persist (Kirkpatrick and Barton 2006) and thereby could potentially constitute genomic constraints in hybrid species. Moreover, depending on the type of selection acting on the parent species' phenotypes, hybrid morphology is expected to be more or less restricted. For traits under stabilizing selection in parents, hybrids are expected to be more free to evolve toward a variety of different potential fitness optima, even those extending beyond those of the parents (Bailey et al. 2013). However, when directional selection has contributed to parent species differences, hybrid phenotypes are predicted to be intermediate of the parent taxa and restricted to evolve along the axis of divergence between them (Bailey et al. 2013). Directional selection on parent species could facilitate convergence toward parental phenotypes (Bailey et al. 2013). In this situation, populations of hybrid species could be restricted to trait values reflecting the relative proportion of the genome inherited from the parent species, depending on genomic background, linkage, and constraints associated with genomic architecture.

Hybrid populations differing in genomic composition can be divergent because of genomic contingencies where allele frequencies are most strongly affected by the selection for compatibility with the rest of the genome (Baack and Rieseberg 2007; Eroukhmanoff et al. 2017). Alternatively, populations can be divergent because they have adapted to local selection pressures if the ecological selection is strong enough to cause the locally most adaptive alleles to increase in frequency, in spite of potential opposing genomic background selection. If genomic contingencies are important, we predict an association between the proportion of the genome inherited from one parent species and the phenotypic similarity to that parent species. In the absence of contingencies, populations experiencing the same selection pressures are expected to develop similar phenotypes (Grenier and Greenberg 2005; Ravinet et al. 2012; Runemark et al. 2014, 2015). If contingencies are not important, we expect ecolog- ical factors to best explain phenotypic variation between genomically differentiated populations. Tests for the presence of such genomic contingencies in hybrid species have, however, rarely been made.

Here, we address the relative importance of genomic contingencies and ecology for hybrid phenotypes through testing the predictions outlined above. We investigate how diet, climate, and genomic composition affect beak shape and size in a hybrid species. Our study species, the Italian sparrow, is a homoploid hybrid resulting from interbreeding between the Spanish sparrow (Passer hispaniolensis) and the house sparrow (Passer domesticus; Elgvin et al. 2011; Hermansen et al. 2011; Trier et al. 2014). Beak morphology is affected by both diet (Benkman 2002, 2016) and thermoregulation and climate (Allen 1877; Symonds and Tattersall 2010). As beak morphology differs with dietary niche in commensal and noncommensal subspecies of the house sparrow (Riyahi et al. 2013), it is likely to be influenced by dietary niche in the Italian sparrow as well. Moreover, the recent shift to an anthropogenic niche by the house sparrow could potentially have caused directional selection in one parent species (cf. Riyahi et al. 2013), a scenario under which genomic contingencies in the hybrid species are more likely to arise (Bailey et al. 2013). To be able to address whether there are genomic contingencies from the relative proportion of parental alleles affecting beak morphology, we use three island populations of Italian sparrow from each of the islands Crete, Corsica, and Sicily that show strong differences in genomic composition and appear to represent independent hybridization events (Runemark et al. 2018b). These populations are allopatric from both parent species, and we find no indications of gene flow between these spatially widely separated populations (Runemark et al. 2018b). In line with the predictions outlined above, we expect beak size and shape to correlate with local diet or climate measures if there is strong ecological selection on the beak (Grenier and Greenberg 2005) and genomic contingencies are not important in the system. On the other hand, if contingencies are important, we predict that island origin (reflecting genomic composition) better explains beak morphology. Finally, an interaction between these factors could mold beak shape. Diet (Grant and Grant 1996; Neto et al. 2016), climate (Eroukhmanoff et al. 2013b; Gardner et al. 2016), song (Huber and Podos 2006; Badyaev et al. 2008), and phylogenetic inertia (Shao et al. 2016) have previously been found to affect beak morphology, although the phylogenetic inertia is not likely to be relevant for within-species divergence. Heritability of beak morphology is very high in the house sparrow, with a heritability of $0.98 \pm 0.19 \mathrm{SE}$ for beak length and $0.76 \pm 0.35 \mathrm{SE}$ for beak depth (Jensen et al. 2003). Beak morphology heritability is also high for flycatchers (Merilä and Sheldon 2000), and several studies in Darwin's finches have identified large effect genes underlying beak morphology differences in hybrid species (Lamichhaney et al. 2015). 
Taken together, this strongly suggests a large genetic component in beak morphology variation, but we cannot exclude that phenotypic plasticity (Wund 2012) could contribute to differences between populations. If plasticity is the sole mechanism affecting beak morphology, no contingencies in the form of correlations between proportion of parental alleles in the genome and similarity to the parent species in beak morphology are expected. In the other extreme case, if contingency fully explains differences, we would expect the proportion of parent alleles to be perfectly predictive of similarity to parent phenotype, regardless of any local factor. Predictions for plasticity are clearly different from those of genomic contingencies, but it is not possible to rule out that locally adaptive beak morphology might partly be explained by phenotypic plasticity. An entirely plastic response is highly unlikely, given that we find evidence for elevated differentiation and signals of selection on FGF10 (a candidate gene involved in beak morphology) between these populations (Runemark et al. 2018b).

The effects of climate and diet on beak morphology have not been studied in genomically divergent populations of a hybrid species. Investigating these factors jointly will shed light on whether population differences within hybrid species could be adaptive or may be restricted to values along the axis of parental divergence. Investigating the extent of sexual dimorphism may shed light on the extent of competition and resource partitioning between the sexes (cf. Bolnick and Doebeli 2003; Cooper et al. 2011). The extent of sexual dimorphism may also reflect whether there is male-specific selection on song (cf. Huber and Podos 2006). We used stable isotopes as a proxy for diet, a set of climatic variables previously shown to influence beak size in the Italian sparrow (Eroukhmanoff et al. 2013b), and whole genome estimates of relative parental proportions from an earlier study on the island populations (Runemark et al. 2018b) to address which factors shape phenotypic variation in a hybrid species.

\section{Material and Methods}

We sampled three populations of Italian sparrows from each island of Crete, Corsica, and Sicily during spring 2013 (fig. 1A). We caught 14-38 birds in each population (for sample sizes and sex, see table A1; tables A1, S1-S6 are available online) using mist netting and took digital pictures of the right side of each birds' head with a Nikon D-500 16.2 megapixel camera. The background was $1-\mathrm{mm}^{2}$ paper, and we ensured that the head of the bird was not tilted. Geometric morphometrics was used to analyze beak shape. We used the thin plate spline-based programs developed by Rohlf (1998) for file conversion (tpsUTIL) and digitization of landmarks (tpsDIG2). Five homologous landmarks were placed on the beak, and we drew an outline with seven equidistant points (i.e., semilandmarks) to further capture beak shape (fig. A1; figs. A1, S1-S5 are available online). PAST (Hammer et al. 2001) was used to estimate relative warps (RWs) and centroid size. The data set used to estimate the RWs included both parental species as well as all Italian sparrow populations included in the study. Relative warps are principal components of shape (Zelditch et al. 2004) and were extracted $(n=32)$ and imported to $\mathrm{R}$ for further analysis. All further statistical analyses were performed in $\mathrm{R}$ ( $\mathrm{R}$ Development Core Team 2013). Because feathers for female stable isotope analysis were sampled for only one population on each island, we performed all tests on two additional data sets to ensure that this did not bias our findings. The two data sets included one set with only one population from each island with both males and females and one set with all nine populations including only males. Sexual dimorphism in beak morphology was small, and the findings based on these data sets were consistent with these from the whole data set (data not shown).

First, we established whether there were significant differences in beak size and shape using centroid size and the four RWs deviating from the noise floor (table S1) as response variables in ANOVA and MANOVA, respectively. We tested both for the presence of overall population variation and for variation among populations within islands using models with population nested within island.

Next, we investigated which factors best explain size and shape variation. We used stable isotopes as a proxy for dietary differentiation. The combination of $\delta^{15} \mathrm{~N}$ and $\delta^{13} \mathrm{C}$ isotope ratios provides a comprehensive picture of diet; $\delta^{15} \mathrm{~N}$ differentiation increases with each trophic level and is indicative of the trophic position in the food web (reviewed in Caut et al. 2009). $\delta^{13} \mathrm{C}$ varies between $C^{3}$ and $C^{4}$ plants (Fry 2006), and $\delta^{13} \mathrm{C}$ ratios in plants decrease with rainfall (Stewart et al. 1995; Ferrio and Voltas 2005); therefore, $\delta^{13} \mathrm{C}$ values are a proxy for dietary source. To obtain stable isotope values, we sent great covert feathers sampled during spring (MarchJune; $1 \pm-0.2 \mathrm{mg}$ finely cut samples in tin capsules; article D1008, Elemental Microanalysis, Devon, UK) for $\delta^{13} \mathrm{C}$ and $\delta^{15} \mathrm{~N}$ analysis at the Stable Isotope Facility (University of California, Davis). Because the aim was to examine population differences, and because sparrows feed on a wide variety of resources, we did not attempt to examine isotopic contents of potential diet items but rather whether diet differed. Because baseline climatic differences could affect isotopic contents, we examined whether values clustered within islands. This was not the case (data not shown), and dietary differences were therefore not overrun by baseline signatures. We also used climatic factors previously shown to correlate with beak size measurements in Italian sparrows (Eroukhmanoff et al. 2013b) as proxies for local climate. We extracted climate variables (annual temperature, annual precipitation, temperature seasonality, and precipitation seasonality) from the WorldClim database (Hijmans et al. 2005); table S3) using the R packages raster (Hijmans and van Etten 
A
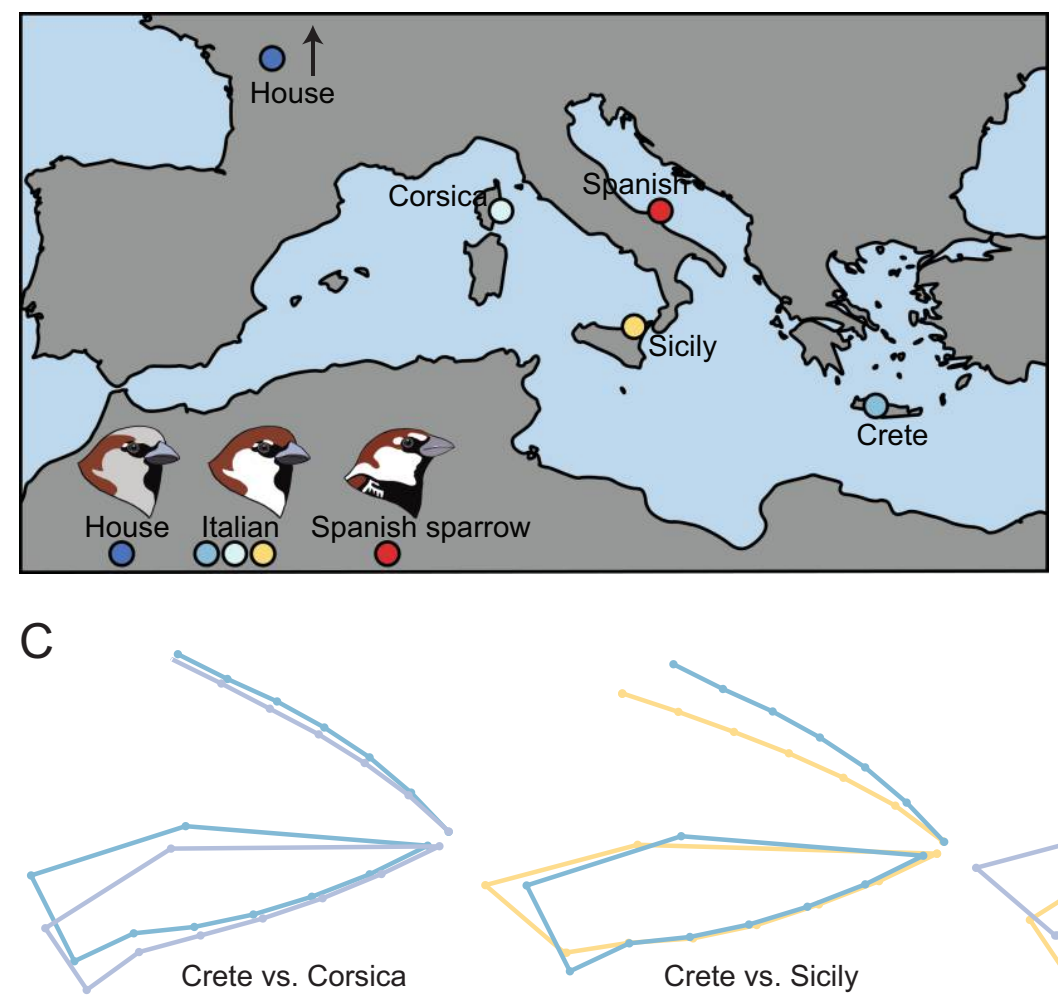

D

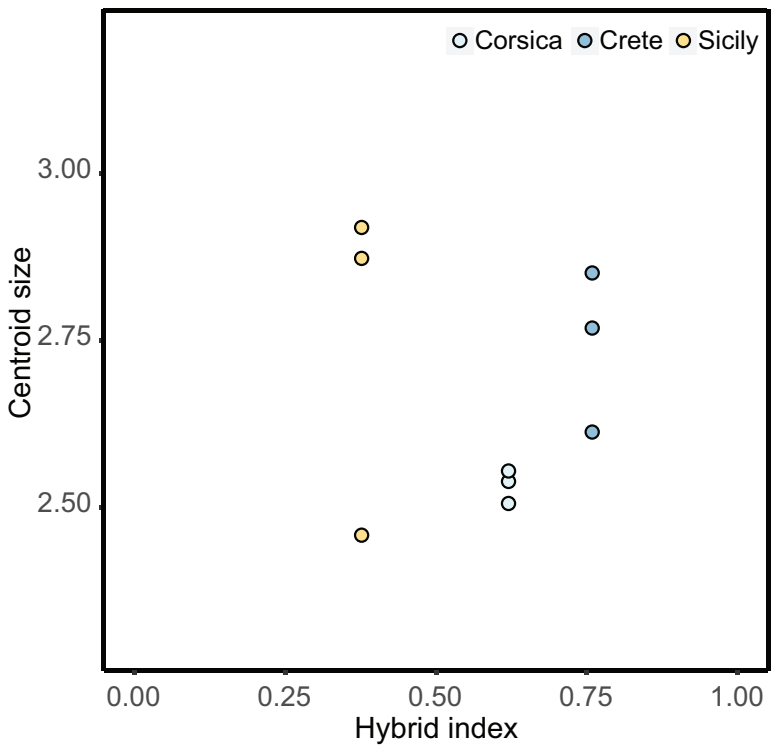

B
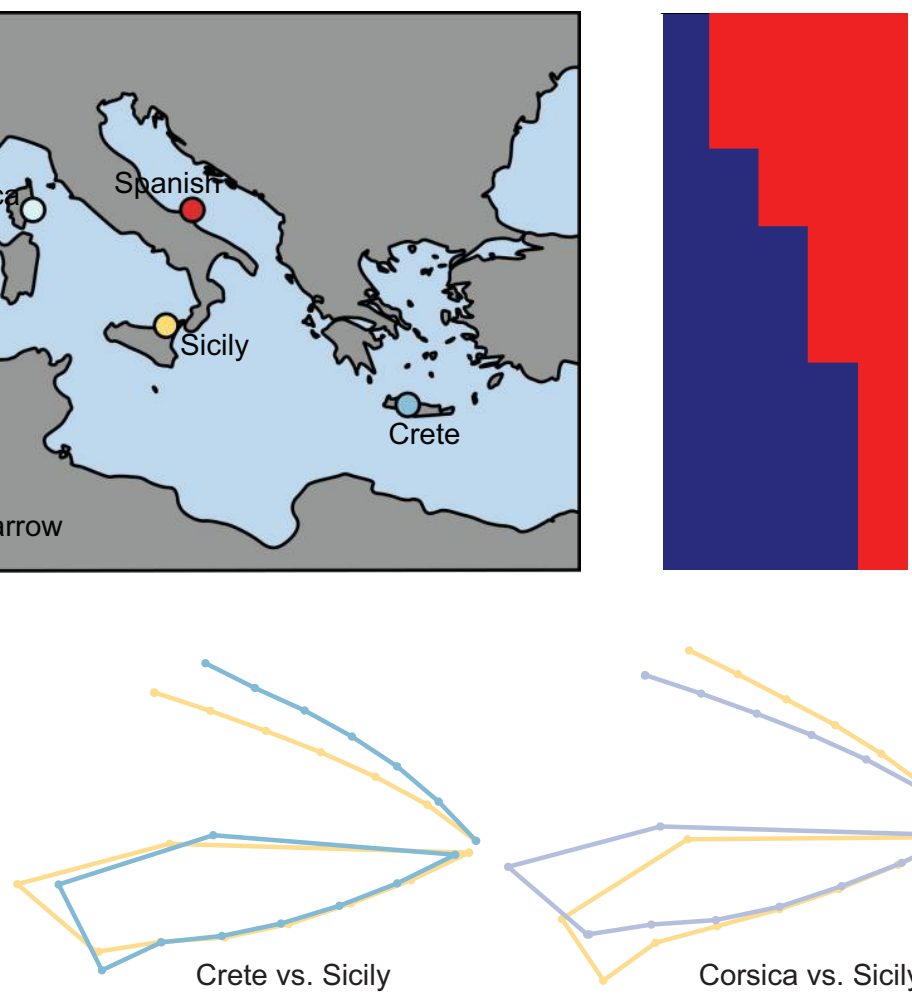

E

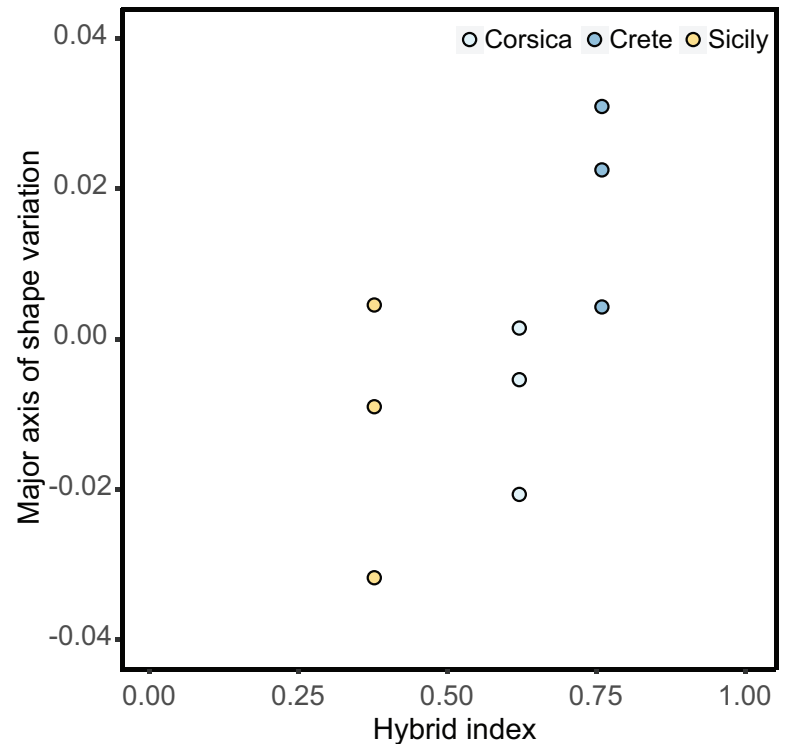

Figure 1: Description of the study system and beak morphology divergence. The Italian sparrow is a hybrid between the house sparrow and the Spanish sparrow. A, Independent, genetically divergent populations are found on the islands of Crete, Corsica, and Sicily (Runemark et al. $2018 b$ ). Three populations were sampled from each island (for coordinates, see table A1, available online). B, Hybrid index (i.e., the probability of house sparrow origin based on whole genome data) differs between populations, with Crete being most house-like and Sicily most Spanish-like. C, Pairwise mean beak shape differences between populations (size differences are scaled). $D$, Population divergence in size is not merely reflecting island of origin. $E$, Major axis of shape variation is not predicted by island of origin either. 
2016), rgdal (Bivand et al. 2016), and foreach (Calaway et al. 2015). Island hybrid index estimates were retrieved from Runemark et al. (2018b), and they show that the Italian sparrow populations on the three studied islands are strongly genetically diverged with independent evolutionary trajectories (Runemark et al. 2018b).

Briefly, for the Muratello (Corsica), Cos (Sicily), and Perama (Crete) populations, Illumina sequence reads were mapped to a repeat-masked version of the house sparrow genome (Elgvin et al. 2017) using BWA 0.7.8 (Li and Durbin 2009) and the BAM file sorted using SAMTOOLS version 1.0 ( $\mathrm{Li}$ et al. 2009). Duplicates were identified and filtered out with MARKDUPLICATES from PICARD-TOOLS version 1.107 (http://broadinstitute.github.io/picard/), indels were identified using RealignerTargetCreator, and local realignments around these were performed with IndelRealigner, both components of GATK 3.3.0 (Van der Auwera et al. 2002; McKenna et al. 2010). Final sequencing coverage of these final BAM files was approximately eight times per individual. Variants were then called using the GATK HaplotypeCaller and then GATK GenotypeGVCFs. This left 49,237,560 variable sites. Indels were first filtered out using VCFtools version $0.1 .12 \mathrm{~b}$ (Danecek et al. 2011), and hard filtering according to the Broad Institute's recommendations was performed with bcftools-1.2 (Li et al. 2009). In addition, we filtered out sites with a mean number of reads per individual less than three, with a genotype quality $<20$ or with a mapping quality lower than 20 using VCFtools version 0.1.12b (Danecek et al. 2011). Genetic admixture was estimated using ADMIXTURE version 0.911 (Alexander et al. 2009). The VCF file was converted to plink's PED format using VCFtools version 0.1.12b (Danecek et al. 2011) and plink version 1.07 (Purcell et al. 2007). Log likelihood values for K, the number of genetic clusters in the data sets, supported two clusters in the data set, and analyses were run for this value. We linkage disequilibrium-pruned the data set; sites within a 50 SNP stepping window with a correlation coefficient higher than 0.1 were omitted. The pruning was performed with plink version 1.07 and left 438,443 sites for analysis. The admixture analysis was performed individually for each island population together with the two parent species. The probability of house sparrow origin was then used as the hybrid index. For a detailed explanation of these genomic methods, see Runemark et al. (2018b). The genomic hybrid index differed between all islands, and within-island genetic differentiation was significant but very low and significantly lower than between island differentiation (table S2). Hence, if beak shape similarity to the parent species corresponds to genomic resemblance, the island hybrid index would be an important factor in the models. Thus, these variables were used as explanatory factors in our models.

To ensure that genetic differentiation within islands was negligible compared with differences between islands, restric- tion site-associated DNA (RAD) sequencing data (A. Cuevas and F. Eroukhmanoff, unpublished manuscript) was used to retrieve within- and between-population $F_{\mathrm{ST}}$. Low withinisland $F_{\mathrm{ST}}$ but with some evidence for local structure would also suggest that allele frequency differentiation and hence local adaptation is feasible at the spatial scale studied.

Centroid size and shape were used as dependent variables. Two models were run for shape: one with only the main axis of divergence (RW1) explaining $>60 \%$ of the variation in shape and another including all four relative warps that deviate from the noise floor. Because climate is identical for all individuals within a population whereas diet may vary between individuals within a population, one populationlevel data set was created to address the effects of both diet and climate, and one individual-level data set was created solely with individual diet estimates. To test which models best explain size and shape, we used a model selection framework based on applicable information criteria.

\section{Population-Level Analyses}

For the population-level analyses, we first tested which ecological factors best explain population divergence in beak size and shape. For the models with centroid size as the dependent variable, corrected Akaike information criterion (AICc) and importance were estimated using the R package MuMIn (Barton 2016). AICc is a version of AIC (Akaike 1974) that is especially suited for small data sets, and importance is the sum of Akaike weights (Wagenmakers and Farell 2004) over all models, including the explanatory variable. The variables with highest importance were used in subsequent models. We then tested which of all possible models best explained data based on AICc, with sex, hybrid index, and their interactions as explanatory variables. The same model was repeated for shape, with the major axis of shape divergence RW1 (reflecting a change from a wide to a narrow basal part of the beak; fig. S1) as a response variable. We also performed a shape analysis including the four main RWs (fig. S1), where selection was based on AIC on MANOVA. Here, we first tested models including only one climate or diet variable and then tested whether adding sex, hybrid index, and/or their interactions improved the model.

\section{Individual-Level Analyses}

For the individual-level data set, model selection was performed as in the population-level analyses but on mixed models, with population as a random factor and with centroid size and RW1 as response variables. We used the lmer command from the R package lme4 (Bates et al. 2016) for these analyses. We first tested which of the ecological variables best explained the model and then explored whether 
adding hybrid index, sex, and/or the interactions improved the model in the same manner as the population-level analyses. To retrieve $F$ and $P$ values for the mixed models, we used the mixed function supplied in the R package afex (Singman et al. 2016).

For the shape analyses, including all four main RWs, the R package MCMCglmm (Hadfield 2010) was used. When the number of groups is low, the posterior distribution of the variance becomes increasingly tail heavy, causing poor mixing of the Markov chain Monte Carlo (MCMC) chain. To mitigate this, we used parameter expansion (Hadfield 2010) on the MCMCglmm algorithm to speed up the rate of convergence in the MCMC chain. This entails using information from a run with an uninformative prior on the same data to choose proper values for the prior means and prior covariance matrix (alpha mean and variance) to be specified in the parameter expanded run. We then used a Cauchy prior as recommended for the parameter expanded run (Hadfield 2010), with the alpha variance set to the square of the standard deviation in the posterior distribution from the uninformative prior. The posterior sampling was run for 200,000 iterations with a burn-in of 40,000 and a thinning of 100. The MCMC chain was plotted and inspected for proper mixing, and autocorrelation remained low $(<0.1)$ between successive samples in the chain. Three chains were run to ensure consistency in parameter estimation. Model selection for these models was performed on the basis of deviance information criterion. To estimate how much of the variation was explained by each variable, variance components were estimated from sum of squares for linear models from the MCMCglmm posterior for multivariate mixed models and with the VCA package version 1.3.3 (Schuetzenmeister and Dufey 2017) for mixed models with univariate response variables.

Finally, we addressed whether the variation among Italian sparrow populations is aligned with the axis of parental divergence or if the phenotypic values attained deviate from this. We used PAST (Hammer et al. 2001) to estimate RWs and centroid size for a data set including both the Italian sparrow populations and one reference population of each parent species. For size, we used an ANOVA with centroid size from this analysis as a response variable and species as a grouping factor. For shape, we performed a discriminant function analysis based on parental values only in PAST (Hammer et al. 2001) and then transformed RW scores for the Italian sparrow individuals into discriminant scores using the factor loadings of the discriminant axis between parent species. We then tested whether the position along the score axis was affected by hybrid index, thus reflecting a correlation between genomic and phenotypic similarity to the parent species using a linear regression. This will shed light on whether genomic composition constrains phenotypic adaptation within the Italian sparrow.
Results

Sex did not significantly affect beak size or shape and was not included in any of the best models for the data set with both females and males in all populations (table S3); therefore, we proceeded with our analyses using the full data set. There was variation in climate variables among populations within islands as well as between islands (table S4), which makes it possible to disentangle effects from climatic factors from these of genomic composition.

\section{Population Divergence in Hybrid Index, Beak Size, and Beak Shape}

Independent island populations from Crete, Corsica, and Sicily differ in the proportion of the genome inherited from house sparrow (Runemark et al. 2018b; fig. 1B). Beak size varies between populations (size: $F_{8,127}=18.75, P=2 \mathrm{E}-16$; shape: $F_{32,508}=2.81, P=1.05 \mathrm{E}-06$; fig. $\left.1 C-1 E\right)$. These differences persist if population is nested within island for both size (island: $F_{2,127}=22.56, P=4.12 \mathrm{E}-09$; population nested within island: $F_{6,127}=17.48, P=1.13 \mathrm{E}-14$ ) and shape (island: $F_{8,250}=6.94, P=2.97 \mathrm{E}-08$; population nested within island: $F_{24,508}=1.69, P=.022$ ). The presence of significant variation within islands shows that differences do not merely reflect genomic composition (fig. $1 C-1 E$ ) but are influenced by other factors.

\section{Beak Size}

Temperature seasonality was the factor best explaining population divergence in beak size and had $\triangle \mathrm{AICc}$ of more than 6 to the second-best model (tables 1, 2; fig. 2A). Because all individuals in a population experience the same climate, we also tested which factors affect beak size at the individual level, excluding climate variables. The best model for individual variation includes $\delta^{15} \mathrm{~N}$, genomic hybrid index, and the interaction between these factors (fig. $2 B$ ), reflecting that $\delta^{15} \mathrm{~N}$ changes do not affect individual beak size in the same manner across islands. Two models were within $\triangle \mathrm{AICc}$ of 2 of this best model (tables 1,2). One included sex and the interaction between sex and hybrid index in addition to the above-mentioned factors, whereas the other included $\delta^{15} \mathrm{~N}$, genomic hybrid index, sex, and the interaction between genomic hybrid index and sex. Hence, patterns of individual beak size variation are complex, and no clear best explanatory variables emerge.

\section{Beak Shape: The Major Axis of Divergence}

The best model for population divergence along the main axis of shape variation, reflecting a change from a wide to a narrow basal part of the beak (fig. S1), included only $\delta^{13} \mathrm{C}$, and 
Table 1: Model selection table

\begin{tabular}{|c|c|c|c|c|c|c|}
\hline Dependent variable(s) & $\begin{array}{l}\text { Replicated } \\
\text { unit }\end{array}$ & Factor & $\begin{array}{l}\text { Random } \\
\text { factor }\end{array}$ & $\mathrm{AICc}$ & $\begin{array}{l}\text { Importance } \\
\text { AICc }\end{array}$ & DIV \\
\hline \multicolumn{7}{|c|}{ Size analyses at population level: } \\
\hline Size & Population & $\delta^{13} \mathrm{C}$ & None & 3.63 & .085 & \\
\hline Size & Population & $\delta^{15} \mathrm{~N}$ & None & 3.1 & .129 & \\
\hline Size & Population & Annual_Temp & None & 2.3 & .180 & \\
\hline Size & Population & Annual_Prec & None & 2.7 & .155 & \\
\hline Size & Population & Temp_Seas & None & -4 & .840 & \\
\hline Size & Population & Prec_Seas & None & 3 & .135 & \\
\hline Size & Population & Island & None & 7.2 & .019 & \\
\hline \multicolumn{7}{|c|}{ Size analyses at individual level: } \\
\hline Size & Individuals & $\delta^{13} \mathrm{C}$ & Population & -140.6 & $<.01$ & \\
\hline Size & Individuals & $\delta^{15} \mathrm{~N}$ & Population & -142.5 & .0126 & \\
\hline Size & Individuals & $\delta^{15} \mathrm{~N}+\mathrm{HI}+\delta^{15} \mathrm{~N} \times \mathrm{HI}$ & Population & -166.6 & & \\
\hline Size & Individuals & $\begin{array}{l}\delta^{15} \mathrm{~N}+\operatorname{sex}+\mathrm{HI}+\delta^{15} \mathrm{~N} \times \\
\mathrm{HI}+\mathrm{HI} \times \operatorname{sex}\end{array}$ & Population & -165.6 & & \\
\hline Size & Individuals & $\delta^{15} \mathrm{~N}+\mathrm{HI}+\operatorname{sex}+\mathrm{HI} \times \operatorname{sex}$ & Population & -164.6 & & \\
\hline \multicolumn{7}{|c|}{$\begin{array}{c}\text { Univariate shape analyses } \\
\text { at population level: }\end{array}$} \\
\hline RW1 & Population & $\delta^{13} \mathrm{C}$ & None & -42.40 & .737 & \\
\hline RW1 & Population & $\delta^{15} \mathrm{~N}$ & None & -36.00 & .103 & \\
\hline RW1 & Population & Annual_Temp & None & -41.80 & .678 & \\
\hline RW1 & Population & Annual_Prec & None & -36.10 & .106 & \\
\hline RW1 & Population & Temp_Seas & None & -35.70 & .088 & \\
\hline RW1 & Population & Prec_Seas & None & -40.20 & .476 & \\
\hline RW1 & Population & Island & None & -36.00 & .103 & \\
\hline \multicolumn{7}{|c|}{$\begin{array}{c}\text { Univariate shape analyses } \\
\text { at individual level: }\end{array}$} \\
\hline RW1 & Individuals & $\delta^{13} \mathrm{C}$ & Population & -337.0 & .176 & \\
\hline RW1 & Individuals & $\delta^{15} \mathrm{~N}$ & Population & -311.9 & $<.01$ & \\
\hline RW1 & Individuals & $\delta^{13} \mathrm{C}+\mathrm{HI}$ & Population & -335.8 & & \\
\hline RW1 & Individuals & $\delta^{13} \mathrm{C}+\operatorname{sex}$ & Population & -334.9 & & \\
\hline \multicolumn{7}{|c|}{$\begin{array}{l}\text { Multivariate shape analyses } \\
\text { at population level: }\end{array}$} \\
\hline RW $1-4$ & Population & $\delta^{13} \mathrm{C}$ & None & -240.1 & & \\
\hline RW1-4 & Population & $\delta^{15} \mathrm{~N}$ & None & -231.1 & & \\
\hline RW1-4 & Population & Annual_Temp & None & -239.5 & & \\
\hline RW1-4 & Population & Annual_Prec & None & -243.5 & & \\
\hline RW1-4 & Population & Temp_Seas & None & -239.4 & & \\
\hline RW1-4 & Population & Prec_Seas & None & -237.2 & & \\
\hline RW1-4 & Population & Annual_Prec $\times$ HI & None & -312.4 & & \\
\hline RW1-4 & Population & Annual_Prec + HI & None & -284.1 & & \\
\hline \multicolumn{7}{|c|}{$\begin{array}{c}\text { Multivariate shape analyses } \\
\text { at individual level: }\end{array}$} \\
\hline RW1-4 & Individuals & $\delta^{13} \mathrm{C}$ & Population & & & 5,792 \\
\hline RW1-4 & Individuals & $\delta^{15} \mathrm{~N}$ & Population & & & 5,789 \\
\hline RW1-4 & Individuals & $\delta^{15} \mathrm{~N}+\mathrm{HI}$ & Population & & & 5,790 \\
\hline RW1-4 & Individuals & $\delta^{15} \mathrm{~N}+\delta^{13} \mathrm{C}$ & Population & & & 5,788 \\
\hline RW1-4 & Individuals & $\delta^{15} \mathrm{~N} \times \mathrm{HI}$ & Population & & & 5,792 \\
\hline RW1-4 & Individuals & $\delta^{15} \mathrm{~N} \times \delta^{13} \mathrm{C}$ & Population & & & 5,790 \\
\hline RW1-4 & Individuals & $\delta^{15} \mathrm{~N} \times \delta^{13} \mathrm{C} \times \mathrm{HI}$ & Population & & & 5,792 \\
\hline RW1-4 & Individuals & $\delta^{15} \mathrm{~N}+\delta^{13} \mathrm{C}+\mathrm{HI}$ & Population & & & 5,790 \\
\hline RW1-4 & Individuals & $\delta^{15} \mathrm{~N} \times \delta^{13} \mathrm{C}+\mathrm{HI}$ & Population & & & 5,791 \\
\hline
\end{tabular}

Note: Dependent variable, replicated unit (reflecting whether the analysis was performed at the population or individual level), explanatory factors included in the model, whether a random factor was included, corrected Akaike information criterion (AICc) values, and, when relevant, importance values on which the model selection was based are included. Each set of tests has its own headline, and the best model is presented in bold. DIV, deviance information criterion. 
Table 2: Properties of the best models

\begin{tabular}{|c|c|c|c|c|c|c|}
\hline Dependent variable & Factor & Estimate & $F$ & df & $P / \mathrm{pmcmc}$ & Model $R^{2}$ \\
\hline \multicolumn{7}{|c|}{ Population-level analyses: } \\
\hline Size & Temperature seasonality & .052 & 10.26 & 1,7 & .015 & .59 \\
\hline Warp1 & $\delta^{13} \mathrm{C}$ & & 8.01 & 1,7 & .025 & .47 \\
\hline \multirow[t]{3}{*}{ Warp1-4 } & Annual precipitation & -.002 & 2.78 & 5,3 & .21 & .82 \\
\hline & $\mathrm{HI}$ & .025 & & & & \\
\hline & Annual precipitation $\times \mathrm{HI}$ & $-4.56 \mathrm{E}-05$ & & & & \\
\hline \multicolumn{7}{|c|}{ Individual-level analyses: } \\
\hline \multirow[t]{3}{*}{ Size } & $\delta^{15} \mathrm{~N}$ & -.092 & 2.94 & $1,128.89$ & .09 & .72 \\
\hline & $\mathrm{HI}$ & -1.28 & 2.72 & $1,43.75$ & .11 & \\
\hline & $\delta^{15} \mathrm{~N} \times \mathrm{HI}$ & .16 & 2.89 & $1,128.95$ & .09 & \\
\hline Warp1 & $\delta^{13} \mathrm{C}$ & & $44,774.00$ & 1,29 & .008 & .07 \\
\hline Warp1-4 & $\delta^{15} \mathrm{~N}$ & 8.41 & NA & 1,129 & .0368 & NA \\
\hline
\end{tabular}

Note: Data include $F$ values, degrees of freedom, $P$ values (for $l m$ and lmer models), and pmcmc values (for the MCMCglmm model) and model $R^{2}$ for the models where it is applicable. NA, not applicable.

explained the data significantly better than the second-best model ( $\triangle \mathrm{AICc}>2$; tables 1,2 ; fig. $2 C)$. Individual-level variation in beak shape was also best explained by $\delta^{13} \mathrm{C}$ differences (fig. $2 D$ ), with $\Delta$ AICc to the second-best model of $>4$ (tables 1, 2).

\section{Beak Shape: All Significant Axes of Divergence}

The first four RWs reflecting beak shape variation deviated from the noise floor (fig. S1). The model best explaining this shape variation included annual precipitation, genomic hybrid index, and the interaction between these terms (fig. S2AS2D; tables 1, 2). We also tested which factors affect beak shape at the individual level, excluding climate variables. Individual shape differences were best explained by a model including only $\delta^{15} \mathrm{~N}$ (fig. S2E-S2H; tables 1,2 ). Variance components show that while ecological factors explain a large degree of the variation at the population level, only a small fraction of the variation between individuals is explained by the factors investigated. Variance components for all models are presented in table S5.

\section{Parental Phenotypes and the Extent of Genomic Contingencies}

We estimated the axis discriminating the parent species on the basis of the four RWs deviating from the noise floor (table S6) and scored the hybrids on this axis. We found a significant correlation between hybrid index and score along the parental axis of variation (estimate $=9.08 \pm 2.95, F_{1,199}=$ 9.50, $P=.002, R^{2}=0.05$ ), implying that populations that are genomically similar to house sparrows also have a more house sparrow-like beak shape. Because hybrid index explains only $5 \%$ of the variation, it might not be a major explanation to beak morphology, even if it has a significant effect. Breaking up shape into the individual axes of variation, we find intermediacy and hence potential constraints in only the third and fourth shape components, reflecting shifts from a proximal to a distal placement of landmark 5 and a high upper mandible to a high lower mandible, respectively (see fig. S1). Italian sparrows attain values outside of the parental range for the first and second axis of variation (fig. S3). Centroid size was nearly significantly correlated with hybrid index (estimate $=0.11 \pm 0.058, F_{1,199}=3.64, P=.058$, $R^{2}=0.01$; fig. S4). Data are deposited in the Dryad Digital Repository: https://dx.doi.org/10.5061/dryad.615s9q5 (Runemark et al. 2018a).

\section{Discussion}

Both beak size and beak shape vary significantly between Italian sparrow populations as well as between islands, but there is no strong sexual dimorphism in either size or shape. Consistent with the hypothesis that hybridization allows for adaptive divergence between populations, we find support for the prediction that ecological factors best explain betweenpopulation variation for both beak size and the major axis of beak shape variation. This highlights a new and interesting role for hybridization in generating adaptive divergence within species, and although this pattern may partly be explained by plasticity, we find several lines of evidence indicative of a substantial genetic component. Additionally, we find some evidence consistent with the prediction for genetic contingencies. Although ecological factors best explain beak shape along the major axis of variation, beak shape divergence for all significant axes of variation is significantly affected by genomic hybrid index, reflecting island of origin and potentially contingencies. The fact that there is a correlation between position along the discriminant axis separating the parent species' shape and the genomic similarity to the parent species is also consistent with a role for contingencies. The presence of such contingencies for shape but not size at the 

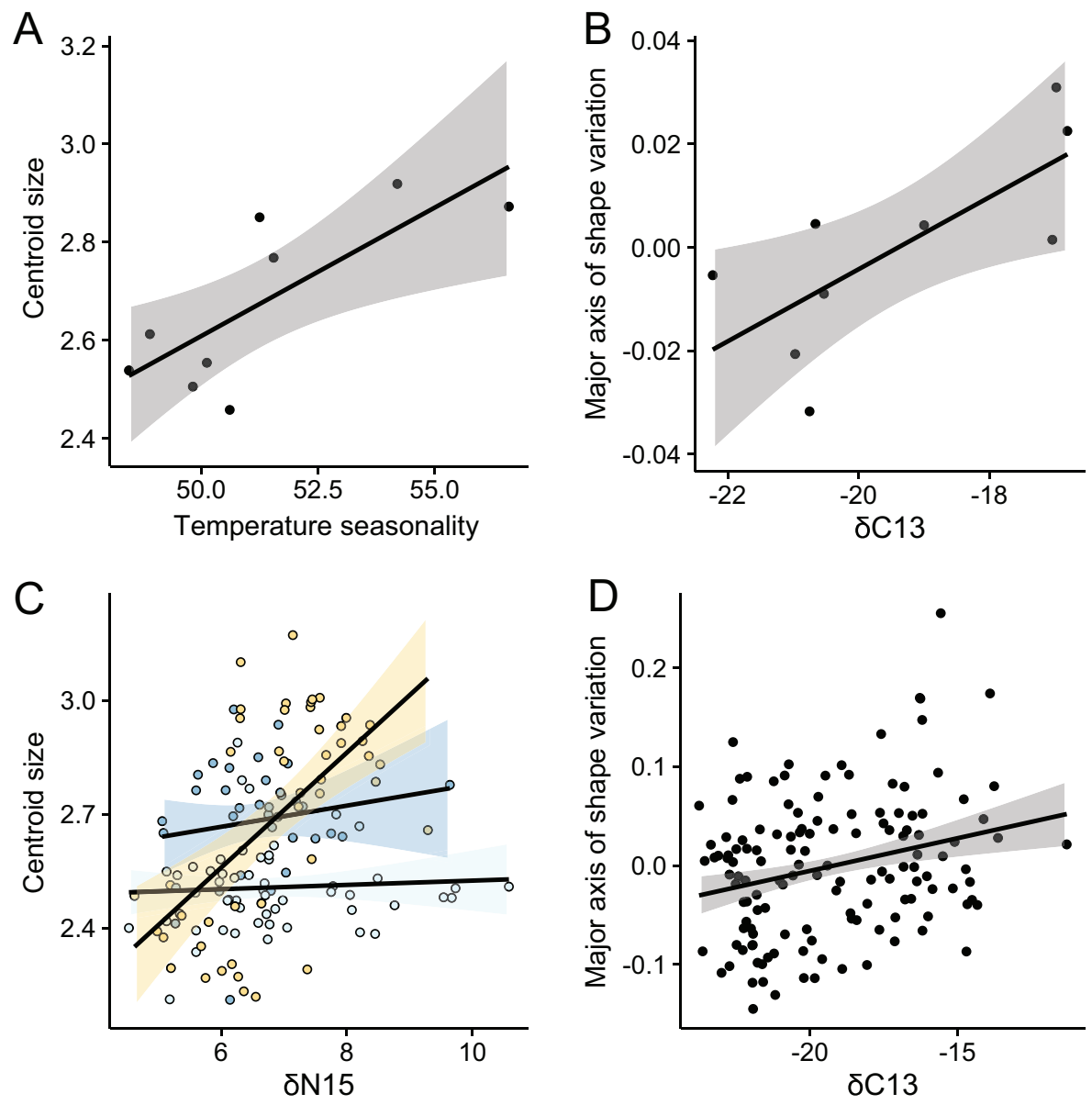

Figure 2: Factors best explaining size and shape variation. $A$, Temperature seasonality is the best predictor of centroid size at the population level, and the relationship is highly significant $\left(F_{1,7}=10.26, P=.015, R^{2}=0.59\right) . B, \delta^{13} \mathrm{C}$ best explained population divergence along the main axis of variation $\left(F_{1,7}=8.01, P=.025, R^{2}=0.47\right)$. $C$, At the individual level, centroid size was best explained by a model including both $\delta^{15} \mathrm{~N}$ and genomic hybrid index and their interaction, as the relationship between $\delta^{15} \mathrm{~N}$ and centroid size varied between islands (model $\left.R^{2}=0.72\right)$. $D$, Individual-level shape divergence along the axis of largest variation was, as for the population level, best explained by $\delta^{13} \mathrm{C}\left(R^{2}=0.07\right)$.

population level is consistent with the finding that shape is less evolvable than size in the fossil record (Hunt 2007). Patterns of individual axes of variation suggest that there may be contingencies in some but not all directions of variation (figs. S3, S4). Overall, these findings suggest that hybrid species phenotypes are shaped by an interplay between selection for a functional genome and a locally adaptive phenotype, and this is an interesting venue for hybridization research.

Interestingly, size and shape are not best explained by the same factors at the population level. While beak size is strongly affected by temperature seasonality, the main axis of beak shape variation is best explained by variation in carbon isotopic ratios. When subjected to strong directional selection pressures, head size and shape may evolve in concert to achieve a function such as strong bite force (Runemark et al. 2015), in contrast to the pattern observed here. There are various reasons temperature regime could affect beak size. Temperature variation could affect the size spectrum of the available diet. There is mounting evidence that beaks play an important role in thermoregulation because blood flow through the network of supportive blood vessels beneath the keratinized surface is augmented at high temperatures and restricted in the cold (Symonds and Tattersall 2010; CampbellTennant et al. 2015). For instance, beak sizes vary as expected from Allen's rule (Allen 1877), which posits that the relative size of body extremities is smaller in colder environments for ectotherms to reduce thermoregulatory costs (Symonds and Tattersall 2010). Even if the effect of smaller beaks cannot explain a high proportion of total heat loss, as in the toucan (Tattersall et al. 2009), using the beak for thermoregulation could potentially be important during summers 
on these arid Mediterranean islands. Furthermore, the fitness advantage of large bill size could differ depending on local temperature profiles and humidity, even in small passerine birds (Gardner et al. 2016). Individual-level divergence is affected by a more complex combination of factors, and no clear best model emerged, although both nitrogen isotopic composition and genomic hybrid index were included in all models. This relationship could therefore be complex and involve many factors of small effect or variables that we have not measured. Individual behavioral plasticity is another possible explanation for the observed pattern (cf. Brown and Robinson 2016), and if such phenotypic plasticity is important, different age groups could possibly differ in beak shapes if they have experienced different climatic conditions.

Annual precipitation pattern is the ecological factor best explaining beak shape. Interestingly, both general beak shape as well as how precipitation patterns affect beak shape are significantly affected by genomic hybrid index. Precipitation patterns could affect seed size (Moles et al. 2005) and the hardness of seeds (Mohamed-Yasseen et al. 1994). Seed size is known to affect beak size evolution in passerines (Grant and Grant 1993), including in sparrows (Riyahi et al. 2013). In addition, beak shape affects bite force (Herrel et al. 2005), and the correlation between annual precipitation and beak shape could reflect adaptation to deal with harder seeds. As the significant genomic hybrid index term and interaction between genomic hybrid index and annual precipitation suggest, there may be a genomic contingency implying that responses to a similar precipitation regime may differ depending on island of origin. The same increase in annual precipitation does not result in the same shape response across the islands. The correlation between genomic similarity to a parent species and shape similarity to that species suggests that this could potentially be due to genomic contingencies.

Nitrogen isotopic composition is the only factor in the model that best explained individual beak shape differences along all dimensions. Differentiation in isotopic composition between a consumer and dietary items is low, predictable, and conserved across trophic levels (i.e., typically $1 \%$ difference; Peterson and Fry 1987). Therefore, it allows for accurate discrimination of dietary contributions from different nitrogen sources (Newsome et al. 2007). Thus, stable isotope signatures may reflect dietary differences in birds, which in turn may also influence beak shape (Neto et al. 2016). Beak specialization for foraging in different selective regimes are well established in birds (Grant and Grant 1996; Benkman 2002, 2016). The Italian sparrow is an opportunistic human commensal species, which feeds on wild seeds, crop plants, and insects. Specialization enabling foraging on prey from different trophic levels or differences in proportions in individual diet within populations could potentially explain the effect of nitrogen isotopic composition on beak shape. Although all sampled individuals were breeding adults, stable isotope composition reflects diet at molt the previous autumn, and the birds could have belonged to different age classes at this point in time.

There could, of course, be unmeasured variables that contribute to beak shape differences. Among the other factors known to cause beak shape differences, phylogenetic inertia (Shao et al. 2016) is unlikely because it is a within-species comparison, and if divergent song preferences (Huber and Podos 2006; Badyaev et al. 2008) would cause beak morphology to differ from the ecological optimum, we could expect sexual dimorphism because males are more frequently vocalizing and are the only sex expressing song motifs (Wang et al. 2014). Because we do not find significant sexual dimorphism, selection on song is probably not strongly affecting the beak shape in these sparrow populations.

Interestingly, one of the genes that was most divergent between Crete and Sicily in a study of the genomic composition of the island populations was FGF10 (Runemark et al. 2018b). This candidate gene for beak shape shown to be important in beak divergence in Darwin's finches (Lamichhaney et al. 2015) is strongly differentiated between the two populations with the largest shape differences. Together, the correlation between ecological differences and beak morphology and the genomic signature of purifying selection (low Tajima's $D$ Runemark et al. 2018b) for this gene associated with beak phenotype in Darwin's finches makes a strong case that the sorting of parental variants allows hybrid species to locally adapt. Regardless, we cannot entirely exclude that population divergence in beak shape is partly driven by plasticity. However, previous results (which include a $P_{\mathrm{ST}}-F_{\mathrm{ST}}$ analysis) suggest that population divergence in beak shape, at least between islands, is driven by selection (Helén 2016). These $P_{\mathrm{ST}}-F_{\mathrm{ST}}$ values were calculated according to the methods applied by Brommer (2011) and Kaeuffer et al. (2012) and do hence not assume that differences between populations are entirely genetic (e.g., as in Merilä 1997). Whole genome data was used for $F_{\mathrm{ST}}$ stimates in the $P_{\mathrm{ST}}-F_{\mathrm{ST}}$ by Helén (2016). Because the RADs with high quality and coverage preferentially map to conserved areas, whole genome data yields higher divergence estimates and will hence give more conservative estimates in the $P_{\mathrm{ST}}-F_{\mathrm{ST}}$ analyses. The $P_{\mathrm{ST}}-F_{\mathrm{ST}}$ approach allows the inference of local adaptation while controlling for different values of heritability and additive genetic variation across populations (Brommer 2011). It is thus possible to make robust conclusions about the role of selection in driving phenotypic divergence, irrespective of the extent to which the phenotypic trait is plastic. Here, we show that across island populations, irrespective of the degree to which beak shape is heritable, divergence has been driven by selection in many instances as $P_{\mathrm{ST}}$ exceeds $F_{\mathrm{ST}}$ irrespective of the $\mathrm{c} / \mathrm{h}^{2}$ ratio (fig. S5). This pattern is consistent with previous findings in populations on mainland Italy (Eroukhmanoff et al. 2013b). Moreover, there is population structure within islands, even 
if this differentiation is lower than that between islands (table S2), illustrating that allele frequencies differ between these populations and hence could allow to some extent for at least locally adaptive frequencies of alleles for beak morphology.

The island populations of Italian sparrow from this study have contingencies in the proportion of inheritance from each parent species (Runemark et al. 2018b), resulting from mosaic patterns of parental inheritance or conditions during initial hybridization and genome stabilization (cf. Eroukhmanoff et al. 2013a). We find that hybrid taxa are intermediate between parent species for both size and shape, although not for all shape components, because means for the major axis of divergence (RW1) are outside of the 95\% confidence interval of the parent species for both Sicily and Crete (fig. S3a), and RW2 tends to be higher for Crete than any parent species and lower for Sicily (fig. S3b). This is consistent with the pattern predicted for traits, where directional selection contributes to parent species differences in which hybrids are expected to differentiate along the parental axis of divergence (Bailey et al. 2013). Furthermore, the proportion of the parental genome inherited from each species, here measured as hybrid index, was significantly correlated with similarity to the parent species beak shape. Taken together, that genomic hybrid index is involved in the best model explaining population divergence in beak shape and is significantly correlated with position along the parental axis of variation suggests that constraints may affect evolutionary trajectories and evolutionary potential following hybridization. There are, however, two shape dimensions that are transgressive, with Italian sparrows from Sicily and Crete having more negative values for the major axis of shape divergence (RW1) and the Crete population having higher and the Sicily population lower values of the second axis of shape divergence than the parental populations (fig. S3). RW1 reflects a change from a high beak with a low base to a lower beak with a smaller base for higher values, and RW2 reflects a change from a blunt to a more pointed beak shape. The presence of such transgression demonstrates a release of parental constraint for some components of shape and is consistent with the predicted patterns of divergence for traits under stabilizing selection in the parents (Bailey et al. 2013).

In conclusion, this study provides evidence of putative adaptive local divergence within a hybrid species but shows that genomic contingencies could affect the evolutionary potential to respond to selection in a hybrid species. Size and shape divergence are best explained by different selective factors, with temperature patterns affecting size and precipitation patterns and proportion inherited from different parent species predicting shape. Interestingly, we find evidence for constraint in only shape and not size, consistent with patterns in the fossil record suggesting that size is more evolvable than shape (Hunt 2007).

\section{Acknowledgments}

We thank Angelica Cuevas and Mark Ravinet for kindly providing RAD-sequencing data, Bernt-Christian Helén for providing $P_{\mathrm{ST}}-F_{\mathrm{ST}}$ estimates, and Jo S. Hermansen and Maria Tesaker for help with fieldwork. This work was funded by a Wenner-Gren Fellowship to A.R.; a Norwegian Research Council grant to G.-P.S. and A.R.; and Nansenfonden, Kungliga Vetenskapsakademien, Kungliga Fysiografiska Sällskapet i Lund, Helge Ax:son Johnsons Stiftelse, Längmanska kulturfonden samt Lunds Stads djurskyddsfond grants to A.R.

\section{Literature Cited}

Abbott, R., D. Albach, S. Ansell, J. W. Arntzen, S. J. E. Baird, N. Bierne, J. Boughman, et al. 2013. Hybridization and speciation. Iournal of Evolutionary Biology 26:229-246.

Akaike, H. 1974. A new look at the statistical model identification. IEEE Transactions on Automatic Control 19:716-723.

Alexander, D. H., J. Novembre, and K. Lange. 2009. Fast model-based estimation of ancestry in unrelated individuals. Genome Research 19:1655-1664.

Allen, J. A. 1877. The influence of physical conditions in the genesis of species. Radical Review 1:108-140.

Baack, E. J., and L. H. Rieseberg. 2007. A genomic view of introgression and hybrid speciation. Current Opinion in Genetics and Development 17:513-518.

Badyaev, A. V., R. L. Young, K. P. Oh, and C. Addison. 2008. Evolution on a local scale: developmental, functional and genetic bases of divergence in bill form and associated changes in song structure between adjacent habitats. Evolution 62:1951-1964.

Bailey, R. I., F. Eroukhmanoff, and G.-P. Sætre. 2013. Hybridization and genome evolution. II. Mechanisms of species divergence and their effects on evolution in hybrids. Current Zoology 59:675-685. Barton, K. 2016. Package MuMIn. R package 1-63.

Bates, D., M. Maechler, B. Bolker, S. Walker, R. Haubo Bojesen Christensen, H. Singman, B. Dai, G. Grothendieck, and P. Green. 2016. Package lme4. R package 1-113.

Benkman, C. W. 2002. Divergent selection drives the adaptive radiation of crossbills. Evolution 57:1176-1181.

2016. Adaptation to single resources and the evolution of crossbill (Loxia) diversity. Ecological Monographs 63:305-325.

Bivand, R., T. Keitt, B. Rowlingson, E. Pebesma, M. Sumner, R. J. Hijmans, and E. Rouault. 2016. Package rgdal. R package 1-53.

Bolnick, D. I., and M. Doebeli. 2003. Sexual dimorphism and adaptive speciation: two sides of the same ecological coin. Evolution 57:24332449 .

Brommer, J. E. 2011. Whither $P_{\mathrm{ST}}$ ? the approximation of $Q_{\mathrm{ST}}$ by $P_{\mathrm{ST}}$ in evolutionary and conservation biology. Journal of Evolutionary Biology 24:1160-1168.

Brown, A. L., and B. W. Robinson. 2016. Variation in behavioural plasticity regulates consistent individual differences in Enallagma damselfly larvae. Animal Behaviour 112:63-73.

Calaway, R., S. Weston, and R. Analytics. 2015. Package foreach. R package $1-10$

Campbell-Tennant, D. J. E., J. L. Gardner, M. R. Kearney, and M. R. E. Symonds. 2015. Climate-related spatial and temporal variation in bill 
morphology over the past century in Australian parrots. Journal of Biogeography 42:1163-1175.

Caut, S., E. Angulo, and F. Courchamp. 2009. Variation in discrimination factors $\left(\Delta^{15} \mathrm{~N}\right.$ and $\left.\Delta^{13} \mathrm{C}\right)$ : the effect of diet isotopic values and applications for diet reconstruction. Iournal of Applied Ecology 46:443-453.

Cooper, I. A., R. T. Gilman, and J. W. Boughman. 2011. Sexual dimorphism and speciation on two ecological coins: patterns from nature and theoretical predictions. Evolution 65:2553-2571.

Danecek, P., A. Auton, G. Abecasis, C. A. Albers, E. Banks, M. A. DePristo, R. E. Handsaker, et al. 2011. The variant call format and VCFtools. Bioinformatics 27:2156-2158.

Derryberry, E. P., N. Seddon, S. Claramunt, J. A. Tobias, A. Baker, A. Aleixo, and R. T. Brumfield. 2012. Correlated evolution of beak morphology and song in the Neotropical woodcreeper radiation. Evolution 66:2784-2797.

Dittrich-Reed, D. R., and B. M. Fitzpatrick. 2012. Transgressive hybrids as hopeful monsters. Evolutionary Biology 40:310-315.

Dumont, E. R., L. M. Dávalos, A. Goldberg, S. E. Santana, K. Rex, and C. C. Voigt. 2012. Morphological innovation, diversification and invasion of a new adaptive zone. Proceedings of the Roval Societv B 279:1797-1805.

Elgvin, T. O., J. S. Hermansen, A. Fjjarzyk, T. Bonnet, T. Borge, S. A. Sæther, K. L. Voje, et al. 2011. Hybrid speciation in sparrows II: a role for sex chromosomes? Molecular Ecology 20:3823-3837.

Elgvin, T. O., C. N. Trier, O. K. Tørresen, I. J. Hagen, S. Lien, A. J. Nederbragt, M. Ravinet, et al. 2017. The genomic mosaicism of hybrid speciation. Science Advances 3:e1602996.

Eroukhmanoff, F., R. I. Bailey, T. O. Elgvin, J. S. Hermansen, A. Runemark, C. N. Trier, and G.-P. Sætre. 2017. Resolution of conflict between parental genomes in a hybrid species. bioRxiv 102970.

Eroukhmanoff, F., R. I. Bailey, and G.-P. Sætre. 2013a. Hybridization and genome evolution I: the role of contingency during hybrid speciation. Current Zoology 59:667-674.

Eroukhmanoff, F., J. S. Hermansen, R. I. Bailey, S. A. Sæther, and G. P. Sætre. 2013b. Local adaptation within a hybrid species. Heredity 111:286-292.

Ferrio, J. P., and J. Voltas. 2005. Carbon and oxygen isotope ratios in wood constituents of Pinus halepensis as indicators of precipitation, temperature and vapour pressure deficit. Tellus 57B:164-173.

Fry, B. 2006. Stable isotope ecology. Springer, New York.

Gardner, J. L., M. R. E. Symonds, L. Joseph, K. Ikin, J. Stein, and L. E. B. Kruuk. 2016. Spatial variation in avian bill size is associated with humidity in summer among Australian passerines. Climate Change Responses 3:1-11.

Grant, B. R., and P. R. Grant. 1993. Evolution of Darwin's finches caused by a rare climatic event. Proceedings of the Roval Society B 251:111-117.

Grant, P. R., and B. R. Grant. 1996. High survival of Darwin's finch hybrids: effects of beak morphology and diets. Ecology 77:500-509.

2006. Evolution of character displacement in Darwin's finches. Science 313:224-226.

2008. How and why species multiply. Princeton University Press, Princeton, NJ.

Grenier, J. L., and R. Greenberg. 2005. A biogeographic pattern in sparrow bill morphology: parallel adaptation to tidal marshes. Evolution 59:1588-1595.

Hadfield, J. 2010. MCMC methods for multi-response generalized linear mixed models: the MCMCglmm R package. Journal of Statistical Software 33:1-22.
Hammer, Ø., D. A. T. Harper, and P. D. Ryan. 2001. PAST: paleontological statistics software package for education and data analysis. Paleontologica Electronica 4:1-9.

Helén, B. C. 2016. Phenotypic divergence in a hybrid species: the roles of isolation, drift and selection. MSc thesis. University of Oslo.

Hermansen, J. S., S. A. Sæther, T. O. Elgvin, T. Borge, E. Hjelle, and G.-P. Sætre. 2011. Hybrid speciation in sparrows I: phenotypic intermediacy, genetic admixture and barriers to gene flow. Molecular Ecology 20:3812-3822.

Herrel, A., J. Podos, S. K. Huber, and A. P. Hendry. 2005. Bite performance and morphology in a population of Darwin's finches: implications for the evolution of beak shape. Functional Ecology 19:43-48.

Hijmans, R. J., S. E. Cameron, J. L. Parra, P. G. Jones, and A. Jarvis. 2005. Very high resolution interpolated climate surfaces for global land areas. International Journal of Climatology 25:1965-1978.

Hijmans, R. J., and J. van Etten. 2016. Raster. R package 1-244.

Huber, S. K., and J. Podos. 2006. Beak morphology and song features covary in a population of Darwin's finches (Geospiza fortis). Biological Journal of the Linnean Society 88:489-498.

Hunt, G. 2007. The relative importance of directional change, random walks, and stasis in the evolution of fossil lineages. Proceedings of the National Academv of Sciences of the USA 104:18404-18408.

Hunter, J. P. 1998. Key innovations and the ecology of macroevolution. Trends in Ecology and Evolution 13:31-36.

Jarvis, E. D., S. Mirarab, A. J. Aberer, B. Li, and P. Houde. 2014. Whole-genome analyses resolve early branches in the tree of life of modern birds. Science 346:1320-1331.

Jensen, H., B. E. Sæther, T. H. Ringsby, J. Tufto, S. C. Griffith, and H. Ellegren. 2003. Sexual variation in heritability and genetic correlations of morphological traits in house sparrow (Passer domesticus). Journal of Evolutionary Biology 16:1296-1307.

Kaeuffer, R., C. L. Peichel, D. I. Bolnick, and A. P. Hendry. 2012. Parallel and nonparallel aspects of ecological, phenotypic, and genetic divergence across replicate population pairs of lake and stream stickleback. Evolution 66:402-418.

Kirkpatrick, M., and N. Barton. 2006. Chromosome inversions, local adaptation and speciation. Genetics 173:419-434.

Lamichhaney, S., J. Berglund, M. S. Almén, K. Maqbool, M. Grabherr, A. Martinez-Barrio, M. Promerová, et al. 2015. Evolution of Darwin's finches and their beaks revealed by genome sequencing. Nature 518:371-375.

Lamichhaney, S., F. Han, J. Berglund, C. Wang, M. S. Almén, M. T. Webster, B. R. Grant, et al. 2016. A beak size locus in Darwin's finches facilitated character displacement during a drought. Science 24:284-293.

Li, H., and R. Durbin. 2009. Fast and accurate short read alignment with burrows-wheeler transform. Bioinformatics 25:1754-1760.

Li, H., B. Handsaker, A. Wysoker, T. Fennell, J. Ruan, N. Homer, G. Marth, et al. 2009. The sequence alignment/map format and SAMtools. Bioinformatics 25:2078-2079.

Lucek, K., L. Greuter, O. M. Selz, and O. Seehausen. 2016. Effects of interspecific gene flow on the phenotypic variance-covariance matrix in Lake Victoria cichlids. Hydrobiologia 791:145-154.

Mallarino, R., O. Campas, J. A. Fritz, K. J. Burns, O. G. Weeks, M. P. Brenner, and A. Abzhanov. 2012. Closely related bird species demonstrate flexibility between beak morphology and underlying developmental programs. Proceedings of the National Academy of Sciences of the USA 109:16222-16227.

Mallet, J. 2005. Hybridization as an invasion of the genome. Trends in Ecology and Evolution 20:229-237.

. 2007. Hybrid speciation. Nature 446:279-283. 
McKenna, A., M. Hanna, E. Banks, A. Sivachenko, K. Cibulskis, A. Kernytsky, K. Garimella, et al. 2010. The genome analysis toolkit: a MapReduce framework for analyzing next-generation DNA sequencing data. Genome Research 20:1297-1303.

Merilä, J. 1997. Quantitative trait and allozyme divergence in the greenfinch (Carduelis chloris, Aves: Fringillidae). Biological Journal of the Linnean Societv 61:243-266.

Merilä, J., and B. C. Sheldon. 2000. Lifetime reproductive success and heritability in nature. American Naturalist 155:301-310.

Miraldo, A., and I. A. Hanski. 2014. Competitive release leads to range expansion and rampant speciation in Malagasy dung beetles. Systematic Biology 63:480-492.

Mohamed-Yasseen, Y., S. A. Barringer, W. E. Splittstoesser, and S. Costanza. 1994. The role of seed coats in seed viability. Botanical Review 60:426-439.

Moles, A. T., D. D. Ackerly, C. O. Webb, J. C. Tweddle, J. B. Dickie, A. J. Pitman, and M. Westoby. 2005. Factors that shape seed mass evolution. Proceedings of the National Academv of Sciences of the USA 102:10540-10544.

Neto, J. M., L. de Oliveira Gordinho, B. Vollot, M. Marín, J. S. Monrós, and J. Newton. 2016. Stable isotopes reveal differences in diet among reed bunting subspecies that vary in bill size. Journal of Avian Biology 48:284-294

Newsome, S. D., C. Martinez del Rio, S. Bearhop, and D. L. Phillips. 2007. A niche for isotopic ecology. Frontiers in Ecology and the Environment 5:429-436.

Peterson, B. J., and B. Fry. 1987. Stable isotopes in ecosystem studies. Annual Review of Ecology and Systematics 18:293-320.

Purcell, S., B. Neale, K. Todd-Brown, L. Thomas, M. A. R. Ferreira, D. Bender, J. Maller, et al. 2007. PLINK: a tool set for wholegenome association and population-based linkage analyses. American Journal of Human Genetics 81:559-575.

Ravinet, M., P. A. Prodöhl, and C. Harrod. 2012. Parallel and nonparallel ecological, morphological and genetic divergence in lakestream stickleback from a single catchment. Journal of Evolutionary Biology 26:186-204.

R Development Core Team. 2013. R: a language and environment for statistical computing. R Foundation for Statistical Computing, Vienna.

Rieseberg, L. H. 2003. Major ecological transitions in wild sunflowers facilitated by hybridization. Science 301:1211-1216.

Rieseberg, L. H., M. A. Archer, and R. K. Wayne. 1999. Transgressive segregation, adaptation and speciation. Heredity 83:363-372.

Riyahi, S., Y. Hammer, T. Arbabi, A. Sanchez, C. S. Roselaar, M. Aliabadian, and G.-P. Sætre. 2013. Beak and skull shapes of human commensal and non-commensal house sparrows Passer domesticus. BMC Evolutionary Biology 13:1-1.

Rohlf, J. F. 1998. On application of geometric morphometrics to studies of ontogeny and phylogeny. Systematic Biology 47:147-158.

Roy, D., K. Lucek, R. P. Walter, and O. Seehausen. 2015. Hybrid "superswarm" leads to rapid divergence and establishment of populations during a biological invasion. Molecular Ecology 24:5394-5411.

Runemark, A., M. Brydegaard, and E. I. Svensson. 2014. Does relaxed predation drive phenotypic divergence among insular populations? Lournal of Evolutionary Biology 27:1676-1690.

Runemark, A., L. Piñeiro Fernández, F. Eroukhmanoff, and G.-P. Sætre. 2018a. Data from: Genomic contingencies and the potential for local adaptation in a hybrid species. American Naturalist, Dryad Digital Repository, https://dx.doi.org/10.5061/dryad.615s9q5.
Runemark, A., K. Sagonas, and E. I. Svensson. 2015. Ecological explanations to island gigantism: dietary niche divergence, predation, and size in an endemic lizard. Ecology 96:2077-2092.

Runemark, A., C. N. Trier, F. Eroukhmanoff, J. S. Hermansen, M. Matschiner, M. Ravinet, and G.-P. Sætre. 2018b. Variation and constraint in hybrid genome formation. Nature Ecology and Evolution 2:549-556.

Schluter, D. 2000. The ecology of adaptive radiation. Oxford University Press, Oxford.

2009. Evidence for ecological speciation and its alternative. Science 323:737-741.

Schuetzenmeister, A., and F. Dufey. 2017. Package VCA. R package 1112.

Seehausen, O. 2013. Conditions when hybridization might predispose populations for adaptive radiation. Journal of Evolutionary Biology 26:279-281.

Selz, O. M., R. Thommen, M. E. Maan, and O. Seehausen. 2013. Behavioural isolation may facilitate homoploid hybrid speciation in cichlid fish. Journal of Evolutionary Biology 27:275-289.

Shao, S., Q. Quan, T. Cai, G. Song, Y. Qu, and F. Lei. 2016. Evolution of body morphology and beak shape revealed by a morphometric analysis of 14 Paridae species. Frontiers in Zoology 13:1-10.

Singman, H., J. Westfall, F. Aust, S. Højsgaard, J. Fox, M. A. Lawrence, and U. Mertens. 2016. Package afex. R package 1-45.

Stewart, G. R., M. H. Turnbull, S. Schmidt, and P. D. Erskine. 1995. ${ }^{13} \mathrm{C}$ natural abundance in plant communities along a rainfall gradient: a biological integrator of water availability. Australian Journal of Plant Physiology 22:51-55.

Symonds, M. R. E., and G. J. Tattersall. 2010. Geographical variation in bill size across bird species provides evidence for Allen's rule. American Naturalist 176:188-197.

Tattersall, G. J., D. V. Andrade, and A. S. Abe. 2009. Heat exchange from the toucan bill reveals a controllable vascular thermal radiator. Science 325:468-470.

Trier, C. N., J. S. Hermansen, G.-P. Sætre, and R. I. Bailey. 2014. Evidence for mito-nuclear and sex-linked reproductive barriers between the hybrid Italian sparrow and its parent species. PLOS Genetics 10:e1004075.

Van der Auwera, G. A., M. O. Carneiro, C. Hartl, R. Poplin, G. del Angel, A. Levy-Moonshine, T. Jordan, et al. 2002. From FastQ data to high-confidence variant calls: the genome analysis toolkit best practices pipeline. Wiley, Hoboken, NJ.

Wagenmakers, E.-J., and S. Farell. 2004. AIC model selection using Akaike weights. Psychonomic Bulletin Review 11:192-196.

Wang, G., C. E. Harpole, J. Paulose, and V. M. Cassone. 2014. The role of the pineal gland in the photoperiodic control of bird song frequency and repertoire in the house sparrow, Passer domesticus. Hormones and Behavior 65:372-379.

Wund, M. A. 2012. Assessing the impacts of phenotypic plasticity on evolution. Integrative and Comparative Biology 52:5-15.

Zelditch, M. L., D. L. Swiderski, H. D. Sheets, and W. L. Fink. 2004. Geometric morphometrics for biologists: a primer. Elsevier Academic, New York. 